\title{
Critical roles of TRAIL in hepatic cell death and hepatic inflammation
}

\author{
Shi-Jun Zheng, Pu Wang, Galit Tsabary, and Youhai H. Chen \\ Department of Pathology and Laboratory Medicine, School of Medicine, University of Pennsylvania, Philadelphia, \\ Pennsylvania, USA
}

\begin{abstract}
The TNF-related apoptosis-inducing ligand (TRAIL) induces apoptosis of tumor cells but not most normal cells. Its role in hepatic cell death and hepatic diseases is not clear. In vitro studies suggest that murine hepatocytes are not sensitive to TRAIL-induced apoptosis, indicating that TRAIL may not mediate hepatic cell death. Using two experimental models of hepatitis, we found that hepatic cell death in vivo was dramatically reduced in TRAIL-deficient mice and mice treated with a blocking TRAIL receptor. Although both TRAIL and its death receptor 5 were constitutively expressed in the liver, TRAIL expression by immune cells alone was sufficient to restore the sensitivity of TRAIL-deficient mice to hepatitis. Thus, TRAIL plays a crucial role in hepatic cell death and hepatic inflammation.
\end{abstract}

J. Clin. Invest. 113:58-64 (2004). doi:10.1172/JCI200419255.

\section{Introduction}

Hepatic cell death is the leading cause of fatality in patients with liver diseases. Although the morphological characteristics of dying hepatocytes are well documented, the molecular pathways leading to the death of hepatocytes under various pathological conditions are not well understood. Recent studies suggest that death ligands of the TNF family may play a crucial role. Thus, both FasL and TNF appear to be involved in mediating hepatic cell death in experimental models of hepatitis, and blocking FasL and/or TNF may ameliorate the disease to various degrees (1-4). However, whether and to what degree other death ligands are involved in hepatic cell death are not clear.

The TNF-related apoptosis-inducing ligand (TRAIL) induces apoptosis of tumor cells but not most normal cells $(5,6)$. In humans, TRAIL interacts with at least four membrane receptors that all belong to the TNF receptor family. TRAIL receptor 1 (TRAIL-R1 or death receptor 4) (7) and TRAIL receptor 2 (TRAIL-R2, death receptor 5 , TRICK2, or KILLER) $(8,9)$ have cytoplasmic death domains and can activate both caspases and NF- $\kappa B$ (10). The other two receptors, TRAIL-R3 (DcR1) and TRAIL-R4 (DcR2), have truncated death domains. They are not capable of activating caspase cascade but

Received for publication June 20, 2003, and accepted in revised form November 4, 2003.

Address correspondence to: Youhai H. Chen, 614 BRB-II/III, Department of Pathology and Laboratory Medicine, University of Pennsylvania School of Medicine, 421 Curie Boulevard, Philadelphia, Pennsylvania 19104, USA. Phone: (215) 898-4671; Fax: (215) 573-8606; E-mail: yhc@mail.med.upenn.edu.

Shi-Jun Zheng and Pu Wang contributed equally to this work.

Conflict of interest: The authors have declared that no conflict of interest exists.

Nonstandard abbreviations used: TNF-related apoptosisinducing ligand (TRAIL); death receptor 5 (DR5); concanavalin A (Con-A); mononuclear cell (MNC); alanine aminotransferase (ALT); aspartate aminotransferase (AST). may activate NF-KB and block apoptosis (11). Additionally, osteoprotegerin is a soluble receptor for TRAIL $(11,12)$ that is known to inhibit osteoclastogenesis and increase bone density (13). In mice, only one membrane TRAIL receptor has been identified, which shares the highest sequence homology with human death receptor 5 (DR5) (9). As do humans, mice also have at least two decoy receptors that do not have transmembrane domains (14).

The role of TRAIL in hepatic cell death is controversial. Initial studies using soluble recombinant TRAIL suggest that, unlike TNF and FasL, soluble TRAIL may not induce hepatic cell death in mice and nonhuman primates, although it kills sensitive tumor cells in these animals $(15,16)$. However, when cultured in vitro, freshly isolated human hepatocytes, but not murine hepatocytes, are sensitive to apoptosis induced by soluble TRAIL (17). These observations raise questions regarding the roles of TRAIL in hepatic diseases. Are the functions of TRAIL different from species to species, from in vitro to in vivo systems? Are the functions of TRAIL different from normal tissues to inflamed tissues? And above all, is TRAIL involved in hepatic cell death in vivo, and if so, to what degree? To address these questions, we investigated the roles of TRAIL in hepatitis using mice deficient in TRAIL. We found that TRAIL plays a crucial role in both concanavalin A-induced (Con-A-induced) and Listeria-induced hepatitis, and that blocking TRAIL action in vivo protects mice from these hepatic diseases.
Methods
Mice. Normal BALB/c mice were purchased from The Jackson Laboratory (Bar Harbor, Maine, USA). $\mathrm{TRAIL}^{-/-}$mice were generated by gene targeting as pre- viously described (18) and backcrossed for more than ten generations to $\mathrm{BALB} / \mathrm{c}$ mice. All mice were housed in the University of Pennsylvania animal facilities under pathogen-free conditions. 
Induction and evaluation of Con-A-induced hepatitis. Seven- to eight-week-old male mice were injected via the tail vein with either a single dose $(15-25 \mathrm{mg} / \mathrm{kg}$ of body weight) or multiple doses $(8 \mathrm{mg} / \mathrm{kg})$ of Con-A (Sigma-Aldrich, St. Louis, Missouri, USA). Blood was obtained through orbital plexus bleeding, and alanine aminotransferase (ALT) and aspartate aminotransferase (AST) levels were determined using the Sigma transaminase kit (Sigma-Aldrich).

Histochemistry and TUNEL. Tissues were fixed in 10\% buffered formalin and embedded in paraffin. Tissue sections $(5 \mu \mathrm{m})$ were prepared and stained with $\mathrm{H} \& \mathrm{E}$ or TUNEL as we described (19). The number of apoptotic cells in sections was determined by light microscopy. The total areas of tissue sections were measured using Image-Pro Plus software (Media Cybernetics Inc., Silver Spring, Maryland, USA) in a blinded manner. The degree of apoptosis in the tissue was determined as the number of apoptotic cells divided by the total area of the sections measured. A total of ten tissue sections were analyzed for each animal.

Caspase- 3 assay. To prepare liver extracts, we first homogenized the tissue in hypotonic buffer $(25 \mathrm{mM}$ HEPES [pH 7.5], $5 \mathrm{mM} \mathrm{MgCl} 2,1 \mathrm{mM}$ EGTA, $1 \mathrm{mM}$ phenylmethylsulfonyl fluoride, and $1 \mathrm{mg} / \mathrm{ml}$ leupeptin and aprotinin). Homogenates were then centrifuged at $20,000 \mathrm{~g}$ for 15 minutes. The protein concentration in the supernatant was determined by the Bradford method. Caspase-3 activity was measured with the fluorescent substrate Ac-DEVD-AFC $(10 \mu \mathrm{M})$ according to the manufacturer's instructions (BD Biosciences, San Diego, California, USA). Caspase-3 activity is expressed as units per $50 \mu \mathrm{g}$ of total protein.

Adoptive cell transfer. Liver mononuclear cells (MNCs) were isolated as previously described (20). Briefly, liver tissue was pressed through a stainless steel mesh and

\footnotetext{
Figure 1

$\mathrm{TRAIL}^{-/}$mice are resistant to Con-A-induced hepatitis. Normal $\left(\mathrm{TRAIL}^{+/+}\right.$) and TRAIL-deficient (TRAIL ${ }^{-/}$) BALB/c mice, five per group, were injected once with Con- $\mathrm{A}$ ( $25 \mathrm{mg} / \mathrm{kg}$ of body weight) to induce hepatitis as described in Methods. Mice were sacrificed at different time points after the Con-A injection, and livers were harvested and tested as described in Methods. ( $\mathbf{a}$ and $\mathbf{b}$ ) Livers from TRAIL $^{+/+}$and TRAIL ${ }^{-/-}$mice, respectively, 24 hours after Con-A injection. (c and d) H\&E staining of liver sections from TRAIL ${ }^{+/+}$and TRAIL ${ }^{-/-}$mice, respectively, 24 hours after Con-A injection. The arrowhead indicates massive cell death in the TRAIL ++ liver section. Original magnification, $\times 200$. (e and $\mathbf{f}$ ) TUNEL staining of liver sections from TRAIL ${ }^{+/+}$and TRAIL ${ }^{-/-}$mice, respectively, 24 hours after Con-A injection. The arrowhead indicates an aggregate of apoptotic cells in the TRAIL $+/+$ liver section (apoptotic nuclei are shown in brown). Original magnification, $\times 200$. (g) Numbers of apoptotic cells per square millimeter of liver sections of TRAIL ${ }^{+/+}$and TRAIL $\mathrm{T}^{-/}$ mice 24 hours after Con-A injection. The difference between the two groups is statistically significant $(P<0.0001)$. (h) Caspase- 3 activity in the liver cytosolic protein extract at different time points after Con-A injection. The difference between the two groups is statistically significant $(P<0.01)$. Results shown are from one representative experiment of three.
}

suspended in PBS. After washing, the cells were resuspended in 33\% Percoll solution and centrifuged at 800 $g$ for 15 minutes. MNCs were collected, washed, and injected into the liver of recipient mice as previously described $(21,22)$. A total of $2 \times 10^{7} \mathrm{MNCs}$ per recipient mouse were used. The purity of the cell preparation was tested by flow cytometry, and the percentages of the following cell types were as follows: $\mathrm{CD}^{+}, 16.5 \% \pm$ $0.8 \%$; $\mathrm{CD}^{+}, 6 \% \pm 0.3 \%$; $\mathrm{B} 220^{+}, 36.5 \% \pm 3.8 \%$; granulocytes, $10.2 \% \pm 8.4 \%$; macrophages, $3.7 \% \pm 0.9 \%$; neutrophils, $1.5 \% \pm 0.4 \%$; and NK cells, $0.9 \% \pm 0.1 \%$. Similarly, splenocytes, after removal of erythrocytes by hemolysis, were injected into the spleen of recipient mice as previously described $(21,22)$. A total of $3 \times 10^{7}$ splenocytes per recipient mouse were used. Hepatitis was induced 1 hour after the cell transfer by intravenous administration of Con-A.

Northern blot. Total RNA was extracted from liver using the phenol/chloroform-sarcosyl extraction/LiCl precipitation method. Purified RNA, $10 \mu \mathrm{g}$ per sample, was separated by electrophoresis on a $1.5 \%$ denaturing agarose gel. The fractionated RNA was then transferred onto a Hybond $\mathrm{N}^{+}$membrane (Amersham Pharmacia Biotech, Piscataway, New Jersey, USA), and blotted with
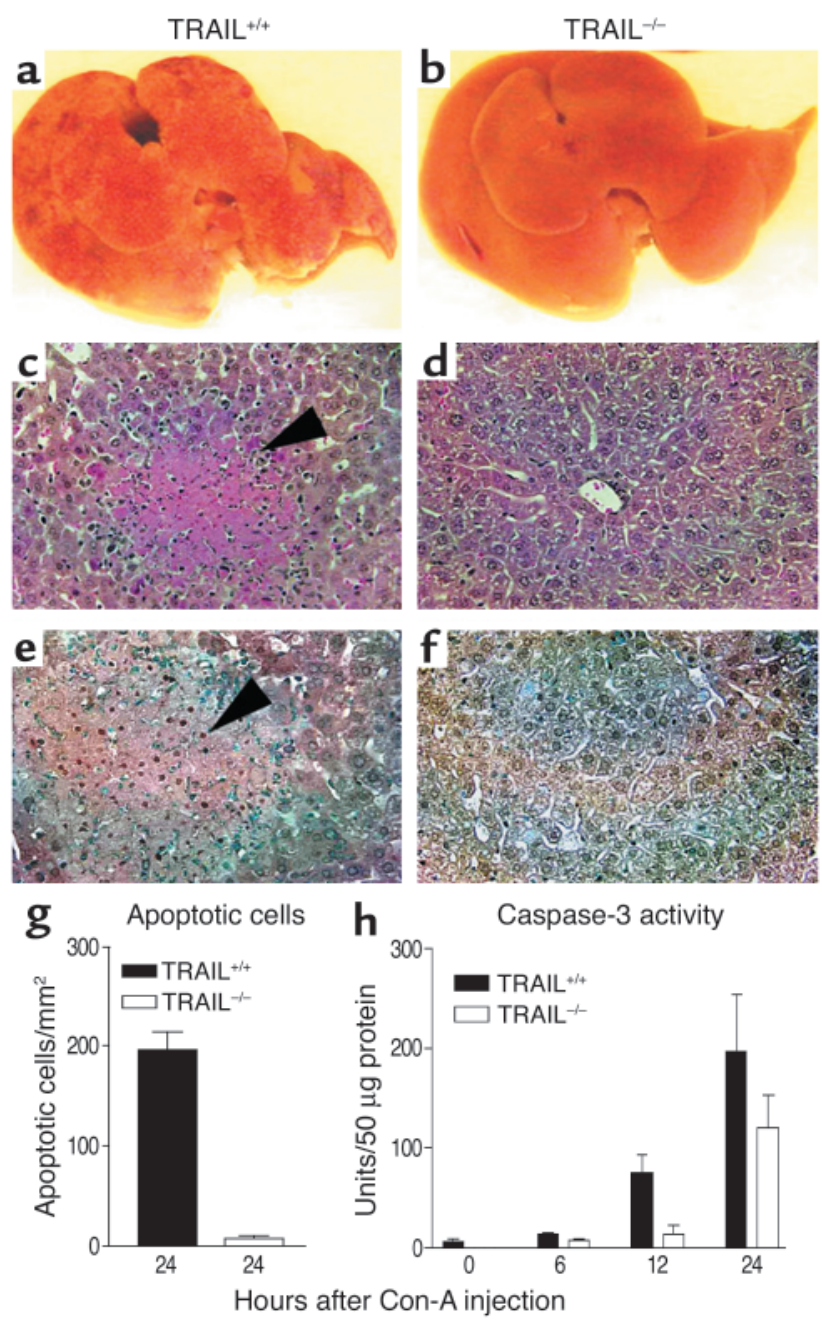

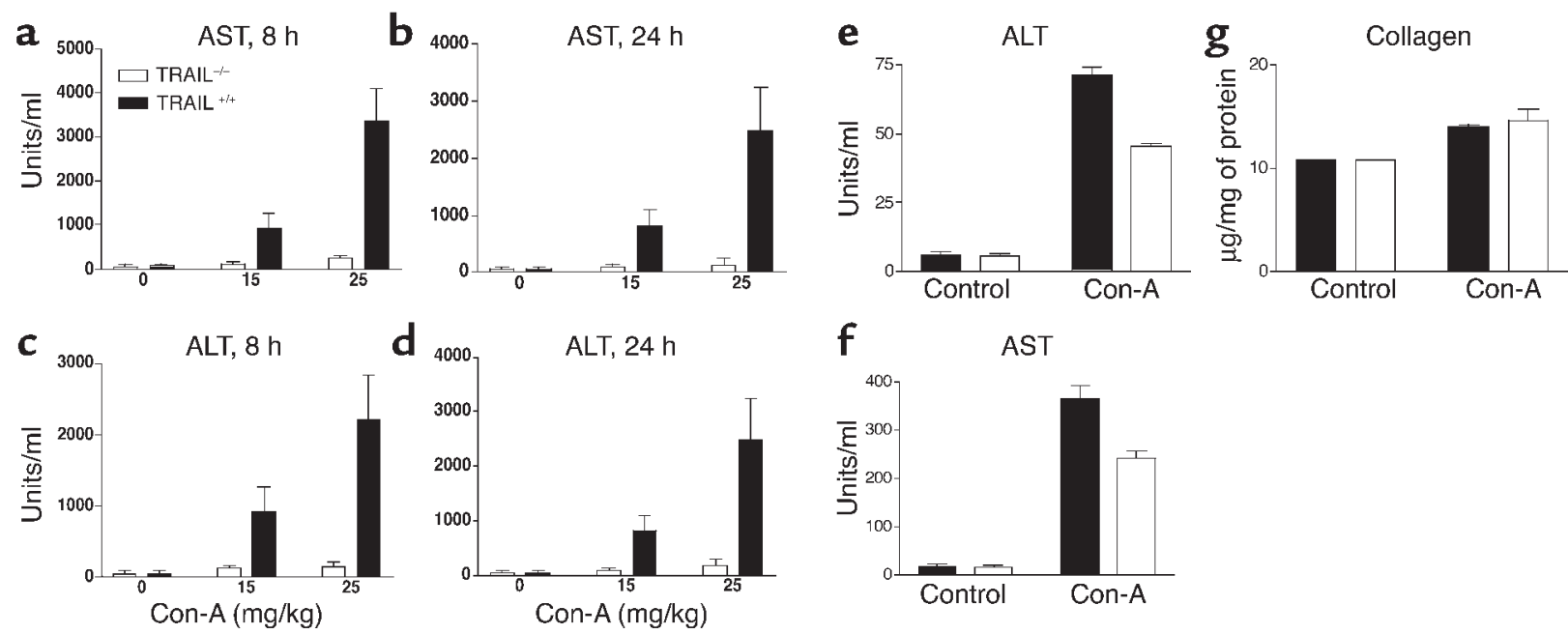

Figure 2

Serum transaminase and liver fibrosis in Con-A-induced hepatitis. (a-d) Normal (TRAIL ${ }^{+/}$) and TRAIL-deficient (TRAIL $/-$) BALB/c mice, five per group, were injected once with Con-A ( $0-25 \mathrm{mg} / \mathrm{kg}$ of body weight) as described in Methods. Serum transaminase levels were determined 8 and 24 hours after the Con-A injection. The ALT and AST levels are presented in Sigma-Frankel units. The differences between the two groups are statistically significant as determined by ANOVA $(P<0.001)$. (e-g) TRAIL ${ }^{-/-}$and TRAIL ${ }^{+/+}$mice, three per group, were injected with a low dose of Con-A ( $8 \mathrm{mg} / \mathrm{kg}$ of body weight) in PBS once a week for 6 consecutive weeks (25). Control mice received only PBS injections. One week after the last injection, mice were sacrificed and serum transaminase activities determined (e and $\mathbf{f})$. The degree of fibrosis was determined by measurement of the amount of collagen per milligram of total liver proteins as previously described (24). The differences between the two groups after Con-A injections are statistically significant $(P<0.05)$ for AST and ALT, but not for collagen.

${ }^{32}$ P-labeled full-length mouse TRAIL, DR5, or GAPDH cDNA probe using the Amersham Pharmacia Biotech Rediprime kit.

Listeria infection. Virulent Listeria monocytogenes were grown in Brain Heart Infusion (Becton Dickinson, Franklin Lakes, New Jersey, USA). Log-phase growing cultures were washed twice with PBS, aliquoted, and stored at $-70^{\circ} \mathrm{C}$ until use. Mice were injected via the tail vein with various CFUs of the bacteria in $200 \mu \mathrm{l}$ PBS. Livers were collected at different time points after $L$. monocytogenes injection, and tissue homogenates were prepared. The CFUs of the inoculum and the organ homogenates were determined by plating of the samples of various dilutions on agar plates, and by counting of the numbers of colonies 24 hours later.

\section{Results}

TRAIL-deficient mice are resistant to Con-A-induced hepatitis. To examine the potential roles of TRAIL in ConA-induced hepatitis, $\mathrm{TRAIL}^{+/+}$and $\mathrm{TRAIL}^{-/-}$mice were first injected intravenously with a high dose of Con-A, and hepatitis was evaluated by anatomical and histological examinations of the liver as well as blood transaminase assay. As shown in Figure 1, $\mathrm{TRAIL}^{+/+}$ mice developed severe hepatitis following Con-A injection, which was characterized by widespread lesions, inflammatory infiltrates, and hepatocyte death. Remarkably, no gross lesions were observed in mice that carried a germ-line TRAIL gene mutation. Although inflammatory infiltrates were also observed in TRAIL $-/-$ mice, the number of apoptotic cells was markedly reduced in these animals as compared with
TRAIL $^{+/+}$mice (Figure 1, e and g). This coincided with a significant reduction of active caspase- 3 in TRAIL T- $^{-/}$ liver (Figure 1h). Similarly, serum transaminase assay revealed significantly higher levels of ALT and AST in $\mathrm{TRAIL}^{+/+}$mice than in TRAIL ${ }^{-/-}$mice (Figure 2).

It was recently reported that activated stellate cells expressed DR5 and underwent TRAIL-mediated apoptosis (23). Because stellate cells play important roles in liver fibrosis, it was suggested that TRAIL might regulate fibrosis by inducing apoptosis of stellate cells (23). To test this possibility, we also injected mice multiple times with a low dose of Con-A. Liver fibrosis was assessed by both collagen assay and histochemistry $(24$, 25). Interestingly, as shown in Figure 2, TRAIL defi-

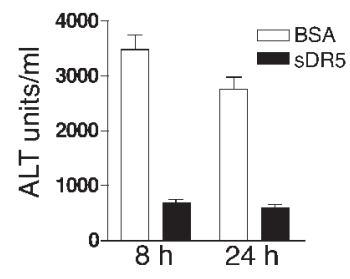

Figure 3

TRAIL blockade protects mice from Con-A-induced hepatitis. $\mathrm{TRAIL}^{+/+} \mathrm{BALB} / \mathrm{c}$ mice, five per group, were injected intraperitoneally with either soluble DR5 (sDR5) or control protein BSA (300 $\mu \mathrm{g} /$ mouse). One hour later, mice were challenged with Con-A (25 $\mathrm{mg} / \mathrm{kg}$ of body weight), and serum ALT activities were assessed 8 and 24 hours after the challenge. Data presented are means and SEM of all mice. Results shown are from one representative experiment of two. The differences between the two groups are statistically significant as determined by ANOVA $(P<0.01)$. 


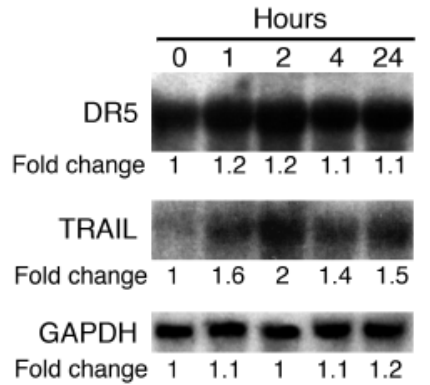

Figure 4

TRAIL and TRAIL receptor gene expressions in Con-A-induced hepatitis. Mice were treated as described in the legend to Figure 1, perfused, and sacrificed at indicated hours after Con-A injection. DR5, TRAIL, and GAPDH gene expressions were determined by Northern blot as described in Methods. Numbers under each lane represent fold changes over signals obtained at 0 hours.

ciency did not appear to significantly affect the degree of fibrosis. Similar results were obtained from histochemical analysis of liver sections following repeated injections of Con-A. However, since hepatitis was less severe in TRAIL $^{-/-}$than in TRAIL $^{+/+}$mice, it is plausible that TRAIL might have inhibited fibrosis in this model. More studies are needed to clarify this issue.

To ensure that the resistance to Con-A-induced hepatitis of TRAIL ${ }^{-/-}$mice is not due to other abnormalities in these animals, we also tested ConA-induced hepatitis in $\mathrm{TRAIL}^{+/+}$mice treated with a blocking soluble TRAIL receptor. Again, normal $\mathrm{TRAIL}^{+/+}$mice developed severe hepatitis as judged by transaminase assay (Figure 3 ) and anatomical and histological examinations (not shown). Hepatitis was significantly ameliorated following a single injection of soluble DR5 (Figure 3). Taken together, these results indicate that TRAIL plays a critical role in the development of Con-A-induced hepatitis.

TRAIL, but not TRAIL receptor, $m R N A$ is increased in the liver during hepatitis. Both TRAIL and TRAIL receptors are constitutively expressed in the liver $(5,6)$. To determine whether their expressions were affected by hepatitis, we performed Northern blot analysis of liver at different time points after Con-A injection. Both TRAIL and DR5 mRNA could be detected in the liver of untreated animals (Figure 4). However, the level of TRAIL expression, but not that of DR5, was significantly increased in Con-A-treated animals. The $D R 5$ mRNA level remained the same throughout the course of this study (Figure 4). This result was also confirmed by PCR analysis using primers specific for murine TRAIL and DR5.

TRAIL expression by MNCs is sufficient to restore Con-Ainduced hepatitis in TRAIL-deficient mice. Lymphoid and myeloid cells of the immune system are initiators and effectors of Con-A-induced hepatitis $(21,22)$. Depleting these cells blocks Con-A-induced hepatitis (21, 22). To investigate whether TRAIL expression by these cells is essential for their pathogenicity in the liver, we performed adoptive cell transfer experiments using purified MNCs from liver and spleen. As shown in Figure 5, when transferred into $\mathrm{TRAIL}^{-/-}$mice, $\mathrm{TRAIL}^{+/+}$, but not $\mathrm{TRAIL}^{-/-}$, MNCs restored the sensitivity of recipient animals to Con-A-induced hepatitis. Both hepatic and splenic TRAIL $^{+/+}$MNCs were effective in restoring hepatitis in TRAIL ${ }^{-/}$animals. These results indicate that TRAIL expression by cells of the immune system is required for the development of Con-A-induced hepatitis.

TRAIL-deficient mice are resistant to Listeria-induced hepatitis. To determine whether the TRAIL effect described above can be generalized to other models of hepatic injury, we also examined the roles of TRAIL in Listeriainduced hepatitis. As shown in Figure 6, Listeria infection induced severe inflammation and tissue injury in the liver of $\mathrm{TRAIL}^{+/+}$mice as judged by histochemistry and serum aminotransferase assay. By contrast, hepatitis was dramatically reduced in $\mathrm{TRAIL}^{-/-}$mice. Larger numbers of apoptotic cells were detected 1 day after $L i s$ teria infection (Figure 6) in $\mathrm{TRAIL}^{+/+}$but not $\mathrm{TRAIL}^{-/}$ mice, which may explain the more severe hepatic injury observed in the former group. Importantly, the numbers of bacteria recovered from different groups at this time point were similar (Figure 7a), although this changed significantly during the late stages of the infection. These results suggest that the differences in hepatocyte apoptosis between $\mathrm{TRAIL}^{+/+}$and TRAIL ${ }^{-/}$ livers at day 1 might not be related to bacteria numbers but were most likely due to the direct involvement of TRAIL in hepatocyte apoptosis.

As expected, TRAIL ${ }^{-/-}$mice survived much better than TRAIL $^{+/+}$mice following infection with lethal doses of Listeria (Figure 7b). However, this may not be due only to differences in hepatitis, since the numbers of Listeria recovered from different groups were also significantly different at late stages of bacteria infection (Figure 7a). Taken together, these results strongly indicate that TRAIL plays crucial roles in Listeria-induced hepatitis.

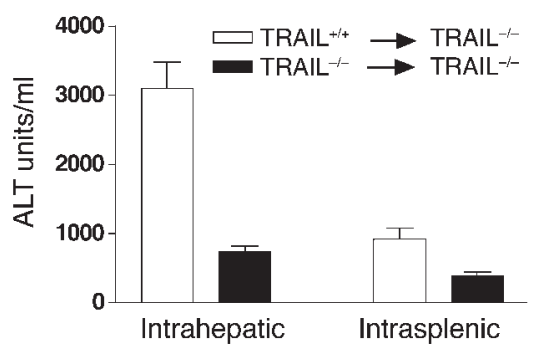

\section{Figure 5}

TRAIL expression by cells of the immune system is required for the development of Con-A-induced hepatitis. TRAIL ${ }^{+/+}$or TRAIL ${ }^{-/}$liver MNCs $\left(2 \times 10^{7}\right.$ cells per mouse $)$ and splenocytes $\left(3 \times 10^{7}\right.$ cells per mouse) were transferred into $\mathrm{TRAIL}^{-/-}$mice intrahepatically and intrasplenically, respectively. One hour later, mice were injected with Con-A ( $25 \mathrm{mg} / \mathrm{kg}$ of body weight), and serum activities of ALT were assessed 12 hours after the challenge. Data presented are means and SEM of each group $(n=5)$. Results shown are from one representative experiment of two. The differences between the two groups are statistically significant as determined by ANOVA $(P<0.01)$. 

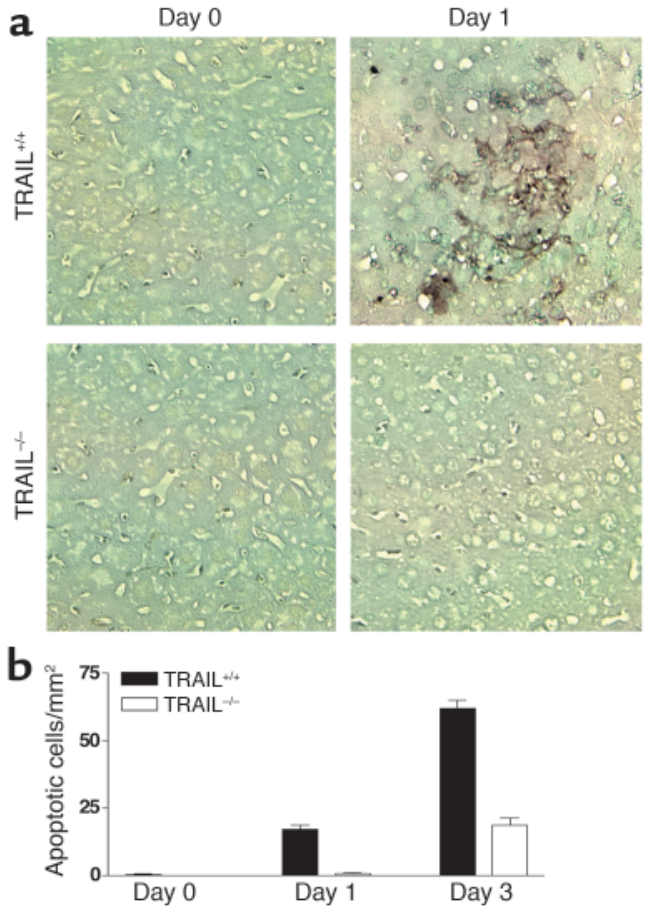
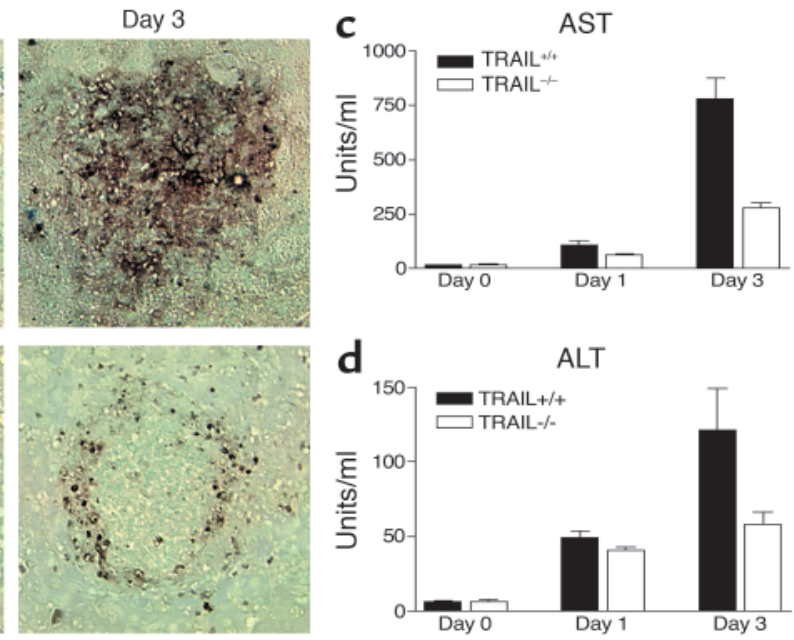

\section{Figure 6}

TRAIL ${ }^{-/-}$mice are resistant to Listeria-induced hepatitis. Groups of TRAIL ${ }^{+/+}$and TRAIL-/- BALB/c mice, five per group, were injected intravenously with $1 \times 10^{4} \mathrm{CFUs}$ per mouse of $L$. monocytogenes. Mice were sacrificed at different times, and their livers were collected and embedded in paraffin. Tissue sections were then prepared and stained by TUNEL as described in Methods. (a) TUNEL staining of liver sections. (b) Quantitative analysis of cell death in the liver. Data presented are means and SEM of each group $(n=5)$. (c and $\mathbf{d})$ Levels of serum transaminases were determined as described in Methods. Results shown are from one representative experiment of four. The differences between the two groups after Listeria infections are statistically significant as determined by ANOVA $(P<0.05)$.

\section{Discussion}

TRAIL holds tremendous promise for cancer therapy because of its ability to selectively induce apoptosis of tumor cells but not most normal cells. However, recent studies using cultured normal cells have cast doubt about this theory. Thus, unlike most normal cells, human hepatocytes, thymocytes, and neural cells appear to be sensitive to TRAIL-induced apoptosis (17, $26,27)$. Results reported here suggest that, in vivo, murine hepatocytes are also sensitive to endogenous TRAIL-induced apoptosis during hepatitis. Blocking TRAIL action in mice ameliorates hepatic cell death and hepatic inflammation. These results are reminis-

\section{Figure 7}

L. monocytogenes counts in the liver and mouse survival rates. (a) Two groups of BALB/c mice $(n=5)$ were treated as described in the legend to Figure 6 and sacrificed 1, 3, and 7 days after Listeria infection. The total number of bacteria in the liver and spleen was determined as described in Methods. Results shown are from one representative experiment of four. The differences between the two groups are statistically significant for days 3 and 7 as determined by ANOVA $(P<0.0001)$. (b) TRAIL $L^{+/+}$ $(n=13)$ and $\mathrm{TRAIL}^{-/-}(n=12) \mathrm{BALB} / \mathrm{c}$ mice were infected with $5 \times 10^{4}$ CFUs of $L$. monocytogenes as described in the legend to Figure 6 . Data shown are survival rates of TRAIL $+/+$ and $\mathrm{TRAIL}^{-/-}$mice pooled from four independent experiments. The difference between the two groups is statistically significant as determined by Mann-Whitney test $(P<0.01)$. cent of reports that TNF and FasL play essential roles in models of hepatitis $(1,2,25)$. Because blocking any one of these death ligands significantly ameliorates hepatitis, they likely play nonredundant roles, and deficiency in one may not be fully compensated for by the others. In the course of our studies, we also exam-
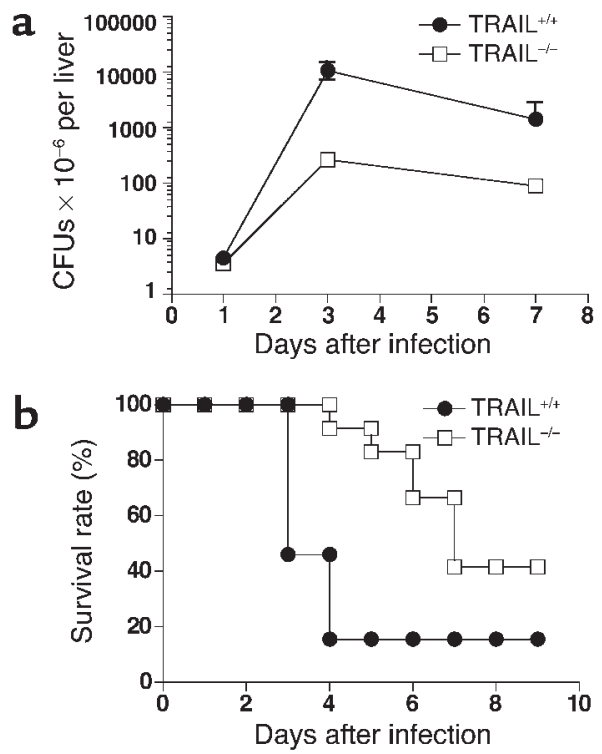
ined the TNF levels in the blood of mice treated with Con-A. We found that TNF was not detectable in the serum of normal BALB/c mice. However, 8-24 hours after a single injection of Con-A $(25 \mathrm{mg} / \mathrm{kg}$ of body weight), significant levels of TNF- $\alpha(25-50 \mathrm{pg} / \mathrm{ml})$ were detected in the serum. Using a similar approach, Ksontini et al. established that TNF- $\alpha$ was directly involved in mediating hepatocyte apoptosis in this model (1). Thus, death of hepatocytes in Con-Ainduced hepatitis is likely mediated by multiple molecular pathways.

It is to be emphasized that experiments described here test directly the roles of endogenous TRAIL (most likely membrane-bound TRAIL) in hepatic cell death in vivo. This is different from other studies in which exogenous TRAIL was administered into animals. Since TRAIL deficiency in our system is global, i.e., it affects all organ systems in mice, it raises the question whether the effects observed on hepatic cell death are due to a direct effect of TRAIL on hepatocytes or an indirect effect of TRAIL on other cells or systems. In the Listeria model, dramatic differences in apoptosis were observed on day 1 when the numbers of bacteria in different animals were the same (Figure $7 \mathrm{~b}$ ). This may rule out the possibility that bacteria are directly responsible for the observed differences in apoptosis. Since no other immunological parameters were different between $\mathrm{TRAIL}^{+/+}$and $\mathrm{TRAIL}^{-/-}$mice at this time point, it is reasonable to conclude that TRAIL is responsible for the difference in hepatic cell death. In the Con-A-induced hepatitis model, differences in apoptosis were observed early and were not associated with other immunological alterations in TRAIL ${ }^{-1-}$ mice. Furthermore, as shown in the adoptive cell transfer experiment (Figure 5), TRAIL expression by MNCs alone is sufficient to restore hepatic cell death in $\mathrm{TRAIL}^{-/-}$animals. Taken together, these results indicate that TRAIL expressed by myeloid and lymphoid cells in the liver is directly responsible for mediating hepatic cell death in hepatitis. This is consistent with recent reports that, unlike soluble TRAIL, membrane TRAIL, when overexpressed in the mouse liver following adenovirus-mediated TRAIL gene transfer, may induce hepatic cell death (28, 29). TRAIL expression is significantly upregulated in patients with hepatitis $B$ virus infection, indicating that TRAIL may indeed play a role in human hepatitis (30).

If TRAIL induces hepatocyte death in hepatitis, why did soluble TRAIL not induce hepatic injury when administered in vivo? It is tempting to speculate that soluble TRAIL may not be able to engage the death receptors in the same manner as membrane TRAIL and may, as in the case of FasL, inhibit membrane TRAIL-induced cell death. The type of TRAIL involved in our model is most likely membrane-bound, although the presence of soluble TRAIL cannot be excluded in the inflamed liver. Additionally, most studies of hepatic cell toxicity of TRAIL were conducted in vitro in the absence of inflammation. In contrast, the experimental system used in this study consists of an inflamed organ whose microenvironment is drastically different from that of normal tissues. Whether the inflammatory condition in the hepatitis animals affects TRAIL function needs now to be investigated.

In addition to inducing apoptosis of hepatocytes and tumor cells, TRAIL may also induce apoptosis of other cell types including stellate cells (23), thymocytes, and neural cells $(26,27)$. Furthermore, it can induce cell cycle arrest of mature $T$ lymphocytes (19). The effects of TRAIL on thymocytes and T cells may explain the heightened autoimmune response in TRAIL-deficient mice (27). Thus, the roles of TRAIL in nontransformed tissues are likely complex, and more investigation is needed before a comprehensive understanding of TRAIL function in vivo can be established. In the meantime, the safety and potential side effects of TRAILbased cancer therapy in humans remain unresolved.

\section{Acknowledgments}

We thank Jacques Peschon (Amgen Inc., Seattle, Washington, USA) for kindly providing the breeders of the TRAIL $^{-1-}$ mice, and Hao Shen for valuable discussions. This work was supported by grants from the NIH (AI50059, AI055934, AI55934, and NS40188).

1. Ksontini, R., et al. 1998. Disparate roles for TNF-alpha and Fas ligand in concanavalin A-induced hepatitis. J. Immunol. 160:4082-4089.

2. Ando, K., et al. 1997. Perforin, Fas/Fas ligand, and TNF-alpha pathways as specific and bystander killing mechanisms of hepatitis $C$ virusspecific human CTL. J. Immunol. 158:5283-5291.

3. Kondo, T., Suda, T., Fukuyama, H., Adachi, M., and Nagata, S. 1997 Essential roles of the Fas ligand in the development of hepatitis. Nat. Med. 3:409-413.

4. Chirmule, N., et al. 1999. Fas-Fas ligand interactions play a major role in effector functions of cytotoxic T lymphocytes after adenovirus vector-mediated gene transfer. Hum. Gene Ther. 10:259-269.

5. Wiley, S.R., et al. 1995. Identification and characterization of a new member of the TNF family that induces apoptosis. Immunity. 3:673-682.

6. Pan, G., et al. 1997. An antagonist decoy receptor and a death domaincontaining receptor for TRAIL. Science. 277:815-818.

7. Pan, G., et al. 1997. The receptor for the cytotoxic ligand TRAIL. Science. 276:111-113.

8. Walczak, H., et al. 1997. TRAIL-R2: a novel apoptosis-mediating receptor for TRAIL. EMBO J. 16:5386-5397.

9. Wu, G.S., Burns, T.F., Zhan, Y., Alnemri, E.S., and El-Deiry, W.S. 1999. Molecular cloning and functional analysis of the mouse homologue of the KILLER/DR5 tumor necrosis factor-related apoptosis-inducing ligand (TRAIL) death receptor. Cancer Res. 59:2770-2775.

10. Schneider, P., et al. 1997. TRAIL receptors 1 (DR4) and 2 (DR5) signal FADD-dependent apoptosis and activate NF-kappaB. Immunity. 7:831-836

11. Degli-Esposti, M.A., et al. 1997. The novel receptor TRAIL-R4 induces NF-kappaB and protects against TRAIL-mediated apoptosis, yet retains an incomplete death domain. Immunity. 7:813-820.

12. Sheridan, J.P., et al. 1997. Control of TRAIL-induced apoptosis by a family of signaling and decoy receptors. Science. 277:818-821.

13. Emery, J.G., et al. 1998. Osteoprotegerin is a receptor for the cytotoxic ligand TRAIL. J. Biol. Chem. 273:14363-14367.

14. Schneider, P., et al. 2002. Identification of a new murine TNF receptor locus that contains two novel murine receptors for tumor necrosis factor-related apoptosis-inducing ligand (TRAIL). J. Biol. Chem. 3:3.

15. Walczak, H., et al. 1999. Tumoricidal activity of tumor necrosis factorrelated apoptosis-inducing ligand in vivo. Nat. Med. 5:157-163.

16. Ashkenazi, A., et al. 1999. Safety and antitumor activity of recombinant soluble Apo2 ligand. J. Clin. Invest. 104:155-162.

17. Jo, M., et al. 2000. Apoptosis induced in normal human hepatocytes by tumor necrosis factor-related apoptosis-inducing ligand. Nat. Med. 6:564.

18. Cretney, E., et al. 2002. Increased susceptibility to tumor initiation and metastasis in TNF-related apoptosis-inducing ligand-deficient mice. J. Immunol. 168:1356-1361. 
19. Song, K., et al. 2000. Tumor necrosis factor-related apoptosis-inducing ligand (TRAIL) is an inhibitor of autoimmune inflammation and cell cycle progression. J. Exp. Med. 191:1095-1104.

20. Bouckson, V., et al. 1994. Characterization of liver lymphomyeloid cells in mice infected with Plasmodium yoelii sporozoites. Immunobiology. 191:413-423.

21. Kaneko, Y., et al. 2000. Augmentation of Valpha14 NKT cell-mediated cytotoxicity by interleukin 4 in an autocrine mechanism resulting in the development of concanavalin A-induced hepatitis. J. Exp. Med. 191:105-114.

22. Takeda, K., et al. 2000. Critical contribution of liver natural killer T cells to a murine model of hepatitis. Proc. Natl. Acad. Sci. U. S. A. 97:5498-5503.

23. Taimr, P., et al. 2003. Activated stellate cells express the TRAIL receptor-2/death receptor-5 and undergo TRAIL-mediated apoptosis. Hepatology. 37:87-95.

24. Gascon-Barre, M., Huet, P.M., Belgiorno, J., Plourde, V., and Coulombe, P.A. 1989. Estimation of collagen content of liver specimens. Variation among animals and among hepatic lobes in cirrhotic rats. J. Histochem. Cytochem. 37:377-381.

25. Song, E., et al. 2003. RNA interference targeting Fas protects mice from fulminant hepatitis. Nat. Med. 9:347-351.

26. Martin-Villalba, A., et al. 1999. CD95 ligand (Fas-L/APO-1L) and tumor necrosis factor-related apoptosis-inducing ligand mediate ischemia-induced apoptosis in neurons. J. Neurosci. 19:3809-3817.

27. Lamhamedi-Cherradi, S.-E., Zheng, S., Maguschak, K., Peschon, J., and Chen, Y.H. 2003. Defective thymocyte apoptosis and accelerated autoimmune diseases in TRAIL ${ }^{-/-}$mice. Nat. Immunol. 4:255-260.

28. Mundt, B., et al. 2003. Involvement of TRAIL and its receptors in viral hepatitis. FASEB J. 17:94-96.

29. Ichikawa, K., et al. 2001. Tumoricidal activity of a novel anti-human DR5 monoclonal antibody without hepatocyte cytotoxicity. Nat. Med. 7:954-960.

30. Han, L.H., et al. 2002. Detection of soluble TRAIL in HBV infected patients and its clinical implications. World J. Gastroenterol. 8:1077-1080 